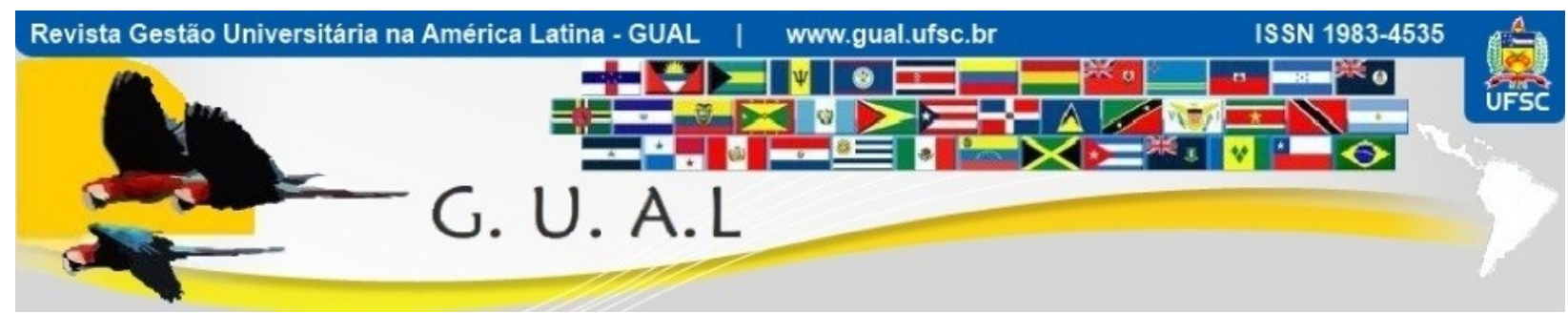

DOI: http://dx.doi.org/10.5007/1983-4535.2012v5n3p1

\title{
O BALANCED SCORECARD COMO FERRAMENTA DE GESTÃO ESTRATÉGICA EM UMA UNIVERSIDADE FEDERAL
}

\section{THE BALANCED SCORECARD AS A STRATEGIC MANAGEMENT TOOL IN A FEDERAL UNIVERSITY}

\author{
Fernando Richartz, Mestre \\ Universidade Federal de Santa Catarina - UFSC \\ nandorichartz@,hotmail.com \\ Letícia Meurer Krüger, Doutor \\ Universidade Federal de Santa Catarina - UFSC \\ ticiamk@hotmail.com \\ Altair Borgert, Doutor \\ Universidade Federal de Santa Catarina - UFSC \\ borgert@,cse.ufsc.br \\ Sergio Murilo Petri, Doutor \\ Universidade Federal de Santa Catarina - UFSC \\ smpetri@gmail.com
}

Recebido em 31/maio/2012

Aprovado em 18/setembro/2012

Sistema de Avaliação: Double Blind Review

Esta obra está sob uma Licença Creative Commons Atribuição-Uso. 


\title{
O BALANCED SCORECARD COMO FERRAMENTA DE GESTÃO ESTRATÉGICA EM UMA \\ UNIVERSIDADE FEDERAL \\ DOI: http://dx.doi.org/10.5007/1983-4535.2012v5n3p1
}

\section{RESUMO}

O objetivo deste trabalho é estruturar um Balanced Scorecard para o departamento de Ciências Contábeis da Universidade Federal de Santa Catarina, cuja finalidade é alinhar uma área de negócio $(\mathrm{CCN})$ com as estratégias definidas pela corporação (UFSC). Para a elaboração do BSC, parte-se das informações contidas no planejamento estratégico do departamento, desenvolvido em 2008, para o período 2009-2020. O mapa estratégico contempla quatro perspectivas, sete subáreas, vinte e seis objetivos, bem como as relações entre eles. A base do mapa são as pessoas, que por meio de processos se relacionam com os clientes e com a sociedade. Posteriormente, com base nos objetivos definidos, elaborou-se o painel balanceado. Nele, cada objetivo possui um indicador de controle, um valor de referência, uma meta (para ser atingida até 2020) e ações ou iniciativas a serem tomadas para atingir os objetivos. No entanto, por serem informações estratégicas, são apresentadas de forma mais detalhada apenas as informações da perspectiva "pessoas". Os resultados dessa perspectiva são: i) Para o indicador "percentual de professores com grupos de estudos registrados no CNPq" tem-se como referência $25 \%$ e estima-se como meta o percentual de $50 \%$; ii) para o percentual de doutores com pós-doutorado concluído ou em desenvolvimento tem-se como referência $20 \%$ e meta $50 \%$; iii) em relação à porcentagem de docentes que cursaram ao menos um curso por ano dos oferecidos pelo Profor tem-se, atualmente, $22 \%$ e, como meta, $80 \%$; iv) para o número de programas Universidade/Professor/Empresa utiliza-se a referência de 2 e meta de pelo menos 5 ; v) por fim, para a porcentagem de professores pertencentes ao CFC, CPC, Ibracon e CRCs tem-se 15\% e pretende-se atingir 30\% até 2020 .

Palavras-chave: Gestores universitários intermediários. Competências. Desenvolvimento de competências gerenciais.

\begin{abstract}
The study's aim is to structure a Balanced Scorecard for the Department of Accounting Sciences of Federal University of Santa Catarina, whose purpose is to align a business area (CCN) with the strategies defined by the corporation (UFSC). To prepare the BSC we depart from the information contained in the strategic planning of the department, developed in 2008 for the period 2009-2020. The strategy map includes four perspectives, seven subareas, twenty-six objectives, as well as the relations between them. The base map is the people, than through processes that relate with customers and the society. After, based on defined objectives, elaborated the balanced panel. In it, each objective has a control indicator, a reference value, an objective (to be achieved by 2020) and actions or initiatives to be taken to achieve the objectives. However, because they are strategic information, are presented in more detail only the information from the perspective "people". The results of this perspective are: i) for the control indicator \% of professors with study groups registered in the CNPQ has $25 \%$ as a reference and it is estimated as a target percentage of $50 \%$, ii) for the $\%$ of doctors with a Pos-Doc completed or under development have as reference $20 \%$ and as target 50 , iii) for the $\%$ of professors who attended at least one course per year offered by the PROFOR has $22 \%$ currently with the target of $80 \%$, iv) for the number of programs University/Professor/Company, reference of 2 and target of at least 5, v) ultimately, for the \% of professors belonging to the CFC, CPC, Ibracon, CRCs the institution now has $15 \%$ and has with target $30 \%$ by 2020 .
\end{abstract}

Keywords: University managers. Competencies. Management development. 


\section{O BALANCED SCORECARD COMO FERRAMENTA DE GESTÃO ESTRATÉGICA EM UMA UNIVERSIDADE FEDERAL \\ DOI: http://dx.doi.org/10.5007/1983-4535.2012v5n3p1}

\section{INTRODUÇÃO}

A função principal de uma universidade é a formação de pessoal qualificado para atuação no mercado de trabalho. Já de acordo com Glat e Pletsch (2004) e Pessoa (2000), a promoção do ensino é a sua função essencial, sua vocação primária. Contudo, para que uma universidade tenha uma boa aplicação dos recursos e, assim, obtenha o retorno esperado, suas ações precisam ser planejadas; com isso, essas ações podem ser mensuradas, de modo que, com base em resultados passados, a instituição possa elaborar estratégias futuras capazes de levá-la a resultados mais eficientes (FREITAS et al., 2010).

Destaca-se que as instituições federais são financiadas com recursos públicos, ou seja, com dinheiro proveniente da população. Diante dessa situação, tais recursos precisam ser gerenciados da melhor forma possível, com a missão de dar para a sociedade um maior retorno - nesse caso, um maior número de profissionais capacitados para atender as demandas do mercado.

Utilizando-se da lógica de Kelm (2003), que comparou as faculdades privadas às empresas em geral, pode-se afirmar que as universidades federais, por possuírem clientes (população), trabalhadores, recursos e estrutura, precisam utilizar ferramentas de gestão que se assemelham às empresas de modo geral. Contudo, para que o propósito dessas instituições seja atingido, é necessário mais que planejamento; é preciso que se execute e controle as ações outrora planejadas (CHIAVENATO, 2003).

Geralmente, as instituições de ensino são avaliadas pelos seus assuntos acadêmicos e pelas suas atividades administrativo-financeiras. Assuntos acadêmicos são monitorados por autoridades externas, por agências de certificação profissional, Secretarias Estaduais de Ensino Superior e órgãos regionais, enquanto a avaliação do resultado para as operações administrativas e financeiras é tratada de forma interna e exige transparência para a sociedade. Além disso, instituições educacionais também têm processos de avaliação interna, conduzidos para serem compatíveis com sua missão e sua visão, no sentido de garantir a melhoria contínua da formação de estudantes, corpo docente e corpo administrativo (KONGAR; PALLIS, 2010).

Assim, tendo em vista a necessidade de gestão estratégica por parte das universidades, o Balanced Scorecard (BSC) pode ser visto como um sistema de gestão que faz a ponte entre os objetivos estratégicos e sua operacionalização. Em outras palavras, ele traduz a visão estratégica em objetivos e medidas capazes de serem avaliadas e comparadas, ao apresentar 


\section{O BALANCED SCORECARD COMO FERRAMENTA DE GESTÃO ESTRATÉGICA EM UMA \\ UNIVERSIDADE FEDERAL \\ DOI: http://dx.doi.org/10.5007/1983-4535.2012v5n3p1}

informações sobre os fatores críticos de sucesso, bem como fornece uma estrutura de comunicação da visão estratégica para os funcionários (CAMPBELL, 1997; KAPLAN; NORTON, 1992; TSENG, 2010). O BSC reflete o plano estratégico da organização em um quadro que permite, a cada pessoa, medir seu desempenho e efetuar ajustes imediatos, se necessários, no intuito do aprimoramento contínuo (CAMPBELL, 1997).

O Balanced Scorecard pode auxiliar a gestão estratégica de duas maneiras complementares: 1) identificando e implementando uma visão estratégica para os objetivos estratégicos e operacionais; 2) facilitando o processo de gestão estratégica (PIENAAR; PENZHORN, 2000). Assim, a avaliação do desempenho de uma universidade é um processo contínuo, que requer monitoramento constante para que se mantenha um alto nível de controle do processo, o qual interfere numa série de aspectos de uma organização. No entanto, existem poucos estudos sobre o desenvolvimento e a implementação do BSC para medir o desempenho das atividades de universidades (TSENG, 2010).

Para que o BSC alcance o êxito desejado pelas organizações, toda a estrutura organizacional deve estar alinhada à estratégia definida no planejamento. Para facilitar esse processo, o BSC corporativo pode ser desdobrado em áreas e segmentos de negócios. Assim, cada área de negócio desenvolve seu próprio $\mathrm{BSC}$, que, por sua vez, alimenta o BSC corporativo e alinha, assim, toda a organização (KAPLAN; NORTON, 2000; KAPLAN; NORTON; RUGELSJOEN, 2010).

Assim, levando-se em consideração a importância das universidades federais para o desenvolvimento da sociedade, e sendo a UFSC a única universidade federal de Santa Catarina a ofertar o curso de Ciências Contábeis, o objetivo deste trabalho é estruturar um Balanced Scorecard para o departamento de Ciências Contábeis da Universidade Federal de Santa Catarina, cuja finalidade é alinhar uma área de negócio $(\mathrm{CCN})$ com as estratégias definidas pela corporação (UFSC) visando sempre o melhor retorno possível para a sociedade.

Utiliza-se o BSC como ferramenta de gestão para o departamento de Ciências Contábeis da Universidade Federal de Santa Catarina pois, mesmo tendo sido concebido originalmente para fins comerciais, a sua abordagem profissional o torna uma ferramenta que se adaqua à configuração de um departamento de ensino (ZBINDEN, 2002). Além do mais, estudos como os de Yu et al. (2009), Kongar e Pallis (2010), Hsieh, Chin e Wu (2004), Chen, Huang e Cheng (2009), Tseng (2010), Rabbani et al. (2010), Zbinden (2002), Pienaar e 


\section{O BALANCED SCORECARD COMO FERRAMENTA DE GESTÃO ESTRATÉGICA EM UMA \\ UNIVERSIDADE FEDERAL \\ DOI: http://dx.doi.org/10.5007/1983-4535.2012v5n3p1}

Penzhorn (2000) e Wachtel, Hartford e Hughes (1999) obtiveram êxito na aplicação do BSC em universidades ou em departamentos de ensino.

Para tratar da temática do BSC, após esta seção de caráter introdutório, apresenta-se o embasamento teórico construído para dar suporte aos resultados deste estudo. Na sequência, abordam-se os aspectos metodológicos, os resultados (a estruturação do BSC) e as conclusões. Por fim, apresentam-se as referências das obras utilizadas como base para esta pesquisa.

\section{FUNDAMENTAÇÃO TEÓRICA}

Desde o início dos anos de 1980, pesquisadores de contabilidade gerencial descrevem a crescente irrelevância do controle e das práticas tradicionais de medição de desempenho. Dentre tais fraquezas, destacam-se a falta de ligação entre medição de desempenho e iniciativas estratégicas das organizações, a ênfase sobre a contabilização de relatórios externos (fiscais e societários) em vez de relatórios contábeis úteis para tomada de decisão interna (gerenciais) e a falta de avanços na tecnologia para mudar a forma como empresas manufatureiras operam.

Nesse cenário, o Balanced Scorecard (BSC) surgiu em 1992, com um estudo realizado por Kaplan e Norton em doze empresas. O BSC complementa o planejamento na medida em que traduz a missão e as estratégias da empresa em um conjunto de indicadores de desempenho estruturados em uma relação de causa e efeito, de modo que esse conjunto serve de base para um sistema de medição e gestão estratégica e possibilita, assim, a implantação, o acompanhamento e a avaliação das estratégias empresariais (GALAS; PONTE, 2006; GUMBUS; LUSSIER, 2006; FREITAS et al., 2010).

O BSC traduz os objetivos (muitas vezes encontrados de forma nebulosa) nos planejamentos corporativos em um roteiro estratégico a ser seguido pelos funcionários. Detalha ações específicas e delineia uma relação de causa e efeito entre as ações e objetivos. O BSC não serve apenas como um sistema de desempenho, mas também como um meio de comunicação de longo prazo de iniciativas estratégicas. Ele ainda combina práticas e conceitos importantes de várias disciplinas e teorias em um único sistema de medição de desempenho, com a finalidade de melhorar o desempenho financeiro (DAVIS; ALBRIGHT, 2004). 


\section{O BALANCED SCORECARD COMO FERRAMENTA DE GESTÃO ESTRATÉGICA EM UMA \\ UNIVERSIDADE FEDERAL \\ DOI: http://dx.doi.org/10.5007/1983-4535.2012v5n3p1}

$\mathrm{Na}$ época do seu surgimento, os conceitos desse instrumento eram mais rudimentares que os atuais. Naquele momento, os autores (Kaplan e Norton) propuseram agrupar os indicadores de desempenho em quatro perspectivas distintas, quais sejam: (a) Financeira, (b) Clientes, (c) Processos Internos e (d) Aprendizado e Crescimento (DIETSCHI; NASCIMENTO, 2008). Um bom Balanced Scorecard, segundo seus idealizadores, deve ser uma combinação adequada de resultados (indicadores de ocorrências) e impulsionadores de desempenho (indicadores de tendências) ajustados à estratégia da unidade de negócio (KAPLAN; NORTON, 1997).

No entanto, o conceito de BSC não é estático. Evidências empíricas sugerem que os usuários do BSC muitas vezes começam com um scorecard simples e rudimentar, que é melhorado em suas funções e em seu escopo de aplicação com o passar do tempo. Infelizmente, isso implica que o Balanced Scorecard está longe de ser um conceito claramente definido (SPECKBACHER; BISCHOF; PFEIFFER, 2003).

Desde os primeiros estudos, em 1992, o BSC vem ganhando crescente popularidade como uma ferramenta de gestão que alinha ações dos funcionários e metas à estratégia corporativa (DAVIS; ALBRIGHT, 2004). Nos últimos anos, o Balanced Scorecard tem atraído interesse considerável tanto na prática como na teoria, e uma vasta gama de literatura sobre ele tem sido publicada em seminários e workshops. Histórias de sucesso de empresas que têm implementado Balanced Scorecards parecem prometer altos benefícios para seus usuários. Várias pesquisas indicam que o conceito BSC é amplamente utilizado em grandes empresas nos Estados Unidos e na Europa (SPECKBACHER; BISCHOF; PFEIFFER, 2003).

O BSC, portanto, parece ser um sucesso global, e tem despertado grande interesse também na Suécia. Um estudo em grandes empresas suecas constatou que $27 \%$ das empresas incluídas na amostra já haviam implementado o BSC (KALD; NILSSON, 2000). Se forem incluídas as empresas que dizem ter o BSC dentro de dois anos, a percentagem sobe para 61\%. Considerando-se que o BSC foi introduzido apenas em 1992, esta deve ser vista como uma propagação rápida e eficaz de uma inovação no campo da contabilidade gerencial. Essa bem sucedida inovação, baseada nos pressupostos ideológicos dos EUA, pode ser atribuída, pelo menos em parte, à forma como a ideia de BSC foi comunicada na Suécia (AX; BJORNENAK, 2005).

Por outro lado, Gumbus e Lussier (2006) afirmam que, embora 50\% das mil maiores empresas da Fortune usem atualmente um Balanced Scorecard, poucas pequenas empresas 


\section{O BALANCED SCORECARD COMO FERRAMENTA DE GESTÃO ESTRATÉGICA EM UMA \\ UNIVERSIDADE FEDERAL \\ DOI: http://dx.doi.org/10.5007/1983-4535.2012v5n3p1}

utilizam essa ferramenta. Assim, o estudo desses autores começa com uma discussão sobre o BSC e discute o porquê de uma pequena empresa usá-lo. Dois estudos de caso são apresentados, com uma cópia de seus BSCs, para ilustrar como a Hyde Park Electronics e a Futura Industries usam um BSC para definir suas estratégias de operações e alinhar suas estratégias para alcançar resultados.

Mesmo com toda essa popularidade, o Balanced Scorecard não tem, no entanto, recebido um acolhimento caloroso na França, onde o Tableau de Bord tem sido usado por pelo menos cinquenta anos. As principais diferenças entre o Balanced Scorecard e o Tableau de Bord podem ser explicadas em termos de pressupostos ideológicos, o que significa que, em grande parte, essas ferramentas de gestão são coerentes com as ideologias locais dos países de origem (BOURGUIGNON; MALLERET; NORREKLIT, 2004).

Contudo, apesar da grande utilização do BSC pelas empresas (principalmente as grandes), pouca atenção é dada a diferentes fatores críticos de apoio, tais como mudança de gestão, gestão de projetos, infraestrutura de desenvolvimento de TI, garantia de qualidade e gestão de riscos, que são críticos para o sucesso na implementação de um Balanced Scorecard (PAPALEXANDRIS et al., 2005). Ao perceberem as dificuldades para as empresas implementarem o BSC, esses autores desenvolveram uma metodologia, baseada em seis etapas, para colocar o BSC em ação: I) Preparar para o projeto; II) Compreender a visão e estratégia; III) Identificar as prioridades estratégicas e objetivos; IV) Selecionar medidas de desempenho; V) Operacionalizar o projeto; e VI) Implementar e acompanhar o sistema.

Em relação às perspectivas do BSC, sugeridas por Kaplan e Norton (1992), encontram-se na literatura tanto trabalhos que as utilizam (a maioria) como outros que as adaptam, o que resulta em perspectivas diferentes. Alguns trabalhos que utilizam quatro perspectivas são: Otley (1999), Lipea e Salterio (2002), Papalexandris, Ioannou e Prastacos (2004), Papalexandris et al. (2005), Gumbus e Lussier (2006) e Braam e Nijssen (2004).

Braam e Nijssen (2004) desenvolveram um estudo que visa contribuir para a compreensão de como usar o BSC de forma eficaz. A pesquisa foi realizada em uma grande instituição financeira holandesa que resolveu implementar o BSC em 1996. A instituição utilizou as perspectivas tradicionais de Kaplan e Norton (1992) e constatou que: a) apesar de sua promessa, gerentes devem estar cientes de que o uso do BSC não implica automaticamente melhora no desempenho da empresa; e b) a tradução da visão e da estratégia em medidas operacionais é um complicado e dinâmico processo. 


\section{O BALANCED SCORECARD COMO FERRAMENTA DE GESTÃO ESTRATÉGICA EM UMA UNIVERSIDADE FEDERAL \\ DOI: http://dx.doi.org/10.5007/1983-4535.2012v5n3p1}

Ainda em relação às perspectivas, à primeira vista, esta parece ser uma abordagem das partes interessadas. Duas das principais áreas definidas representam os principais interessados - nomeadamente, perspectiva financeira e clientes. Outras partes interessadas, como fornecedores, governos, comunidades locais e o meio ambiente, recebem apenas menção passageira (OTLEY, 1999).

Apesar desses dados, existem trabalhos que utilizam, conforme já mencionado, outras perspectivas. Martinsons, Davison e Tse (1999) desenvolveram um Balanced Scorecard para uma empresa de sistemas de informação com as seguintes perspectivas: valor do negócio, orientação do usuário, processos internos e previsão de futuro.

Chavan (2009) escreveu um artigo que busca discutir a importância de sistemas de avaliação de desempenho, em especial o Balanced Scorecard, ao explorar as questões críticas que as organizações enfrentam na construção e implementação dos sistemas, e compartilhou com a comunidade científica as lições aprendidas nas organizações australianas estudadas. $\mathrm{O}$ estudo conclui que a abordagem do Balanced Scorecard pode exigir mudanças substanciais na cultura dentro da organização. Essa ferramenta exige compreensão, compromisso e apoio, desde o nível estratégico até o operacional. Ainda segundo a autora, diferentes organizações têm necessidades, áreas de mercado, pessoas, produtos e serviços completamente diferentes, o que resulta em diferentes perspectivas e, consequentemente, em Balanced Scorecards específicos para cada organização.

Assim, este artigo, levando em consideração os fatores apresentados pela literatura, pretende estruturar um BSC para o departamento de Ciências Contábeis da Universidade Federal de Santa Catarina utilizando-se das quatro perspectivas a seguir (neste artigo, divididas em subáreas): 1) pessoas (corpo docente e técnico-administrativo), 2) processos (coordenação), 3) clientes (discentes e egressos) e 4) sociedade (organizações, concorrentes e MEC).

\section{METODOLOGIA}

O presente estudo consiste em um estudo prático, desenvolvido por meio de uma pesquisa-ação e voltado ao desenvolvimento de um Balanced Scorecard para um gerenciamento mais eficaz do departamento de Ciências Contábeis da Universidade Federal de Santa Catarina. Assim, o estudo envolve a participação efetiva dos pesquisadores e exige ações por parte das pessoas ou grupos envolvidos no problema objeto de estudo. Essa técnica 


\section{O BALANCED SCORECARD COMO FERRAMENTA DE GESTÃO ESTRATÉGICA EM UMA UNIVERSIDADE FEDERAL \\ DOI: http://dx.doi.org/10.5007/1983-4535.2012v5n3p1}

(pesquisa-ação) é muito utilizada para aperfeiçoar programas e para a melhoria da eficácia organizacional e de processos (SOUZA; FIALHO; OTANI, 2007).

Em relação à abordagem do problema, esta pesquisa é qualitativa e os objetivos são tratados de forma descritiva. É qualitativa pois os números aqui utilizados servem apenas de indicadores, e todo o processo, desde sua concepção, necessita de interpretações qualitativas acerca dos fatos. Da mesma forma, é descritiva, pois tem como objetivo primordial a descrição das características de determinada situação encontrada no local de estudo, para posterior estabelecimento de relações entre variáveis (TRIVIÑOS, 1990; GIL, 2007).

As informações utilizadas para o desenvolvimento do BSC são primárias e secundárias. As primárias foram coletadas por meio de entrevistas com os coordenadores de área. Cabe ressaltar que cada subárea possui um coordenador diferente e que algumas possuem dois coordenadores. Já as informações secundárias (negócio, missão, valores, visão, políticas estratégicas, análise de ambientes e fatores críticos de sucesso) foram coletadas no planejamento estratégico 2009-2020 realizado pelos docentes desse departamento em parceria com professores do Instituto de Ensino e Pesquisa em Administração - INEPAD. Algumas informações adicionais necessitaram ser coletadas no site do departamento (CCN, 2011a) e no Plano de Desenvolvimento Institucional (PDI) da UFSC (UFSC, 2011).

Com base nas informações coletadas, estrutura-se o mapa estratégico, no qual são definidos os objetivos de cada subárea. Esses objetivos são fixados com base no planejamento estratégico e estão alinhados a ele. Este foi um processo conjunto entre pesquisadores e coordenadores de área.

Posteriormente, com base nos objetivos definidos no mapa estratégico, estrutura-se um painel balanceado que contem, além dos objetivos de cada subárea, os indicadores de controle, as metas estipuladas, a situação atual (referência) do departamento CCN da UFSC, bem como as ações a serem tomadas para se alcançar as metas propostas. Sempre que possível, para definir as metas, toma-se como base os valores das melhores instituições de ensino. Quando as informações não estão disponíveis, as metas são fixadas com base em parâmetros internos.

As principais limitações deste estudo dizem respeito à interferência dos pesquisadores na definição das variáveis e à confiabilidade das informações secundárias coletadas. Afirmase isso porque é com base nas informações coletadas no planejamento estratégico 2009-2020 que se traçaram os objetivos, metas e indicadores de desempenho. Portanto, para minimizar 


\section{O BALANCED SCORECARD COMO FERRAMENTA DE GESTÃO ESTRATÉGICA EM UMA \\ UNIVERSIDADE FEDERAL \\ DOI: http://dx.doi.org/10.5007/1983-4535.2012v5n3p1}

possíveis falhas, torna-se importante uma revisão, no mínimo anual, do BSC desenvolvido para o departamento de Ciências Contábeis da UFSC.

\section{DESENVOLVIMENTO DO BSC}

O curso de Ciências Contábeis da Universidade Federal de Santa Catarina foi criado no ano de 1965, por meio do Parecer de Criação $n^{0}$ 39, de 8 de fevereiro de 1965, pelo Ministério da Educação e Cultura. O departamento de Ciências Contábeis, o qual atua diretamente nesse curso, se situa no bairro Trindade, em Florianópolis, e conta atualmente com um quadro de 31 professores efetivos, cinco professores temporários (substitutos), três funcionários e três estagiários.

O curso forma bacharéis em Ciências Contábeis, habilitando os formados ao exercício de contador, e possui como objetivo, conforme o currículo de 2006 (CCN, 2011b) "promover a formação do indivíduo como profissional no campo gerencial privado e público, e complementar sua socialização como cidadão brasileiro comprometido com o desenvolvimento econômico e social, no mundo moderno.". Para melhor desempenho das suas atribuições, o departamento está dividido em quatro coordenadorias administrativas: Especialização, Pesquisa e Extensão, Estágio e, por fim, TCC, além da coordenadoria de graduação e de pós-graduação.

Assim, a estruturação do Balanced Scorecard para o departamento de Ciências Contábeis da Universidade Federal de Santa Catarina se baseia na necessidade de operacionalização das ações estabelecidas no planejamento estratégico 2009-2020 dessa unidade. Esse instrumento preserva as medidas financeiras tradicionais e as complementa com medidas dos vetores que impulsionam o desempenho futuro. Seus objetivos e medidas derivam da visão e estratégia da própria organização (ROCHA, 2000).

O planejamento estratégico é um processo de gestão e deve ser desenvolvido em fases, de modo a garantir a obtenção do melhor conjunto de diretrizes estratégicas, capazes de aproveitar as oportunidades e pontos fortes bem como de arrefecer o impacto das debilidades encontradas no ambiente da empresa (CROZATTI, 2003). No planejamento estratégico desenvolvido pelo departamento, foram definidos o negócio, a missão, os valores, a visão, as políticas estratégicas, a análise de ambientes e os fatores críticos de sucesso, conforme se demonstra na figura 1 . 


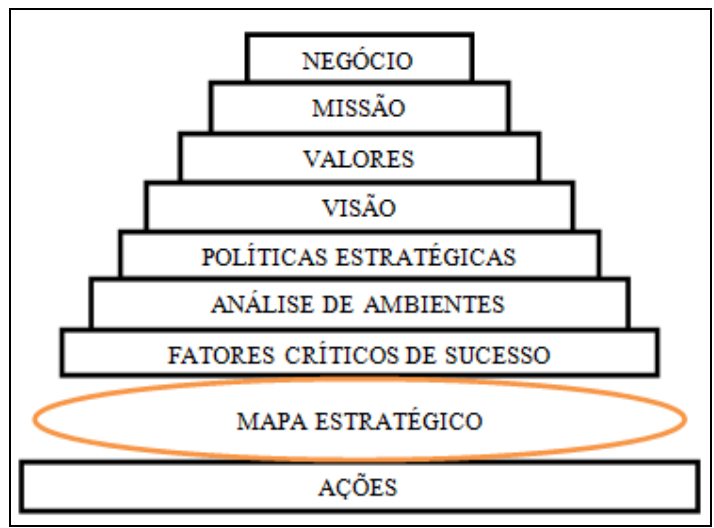

Figura 1 Pirâmide estratégica

De posse dessas informações, busca-se, por meio do BSC, operacionalizar a estratégia com o desenvolvimento do mapa estratégico e de um plano de ações (painel). No planejamento estratégico desenvolvido para o período 2009-2020 (CCN, 2011c), estipularamse as seguintes condições:

$\checkmark$ Negócio: Ser um centro de excelência em ensino, pesquisa e extensão em Ciências Contábeis;

$\checkmark$ Missão: Produzir e difundir os conhecimentos da área contábil, por meio do ensino, pesquisa e extensão, formando profissionais qualificados e, complementarmente, o cidadão como elemento que contribua para o desenvolvimento e a melhoria da qualidade de vida da sociedade;

$\checkmark$ Valores: Promover o ensino de qualidade, priorizando sempre a formação de profissionais éticos e comprometidos com a sociedade;

$\checkmark$ Visão: Ser o melhor curso de Ciências Contábeis do Brasil;

$\checkmark$ Política estratégica: A política adotada pelo departamento é de diferenciação com enfoque na qualidade do ensino;

$\checkmark$ Pontos fortes: Status; gratuidade; referência em conhecimento; professores fortes na pesquisa e no aspecto conceitual; biblioteca com melhor acervo; experiência de parte do corpo docente em organizações públicas e privadas (ensino, pesquisa e extensão); marca UFSC; o fato de ser instituição pública, o que atrai a contratação para diversas atividades por outros órgãos públicos (dispensa de licitação);

$\checkmark$ Pontos fracos: Professores pouco voltados à prática (teóricos); greves (calendários confusos); falta de iniciativa para maior interação com a comunidade, no tocante à pesquisa e extensão; recursos escassos para atualização profissional não formal; falta de visão global das mudanças que estão ocorrendo;

$\checkmark$ Oportunidades: Mais facilidade de emprego; fonte de referência para crescimento acadêmico; mais facilidade para ingressar no mestrado e doutorado; localização geográfica; parque industrial catarinense bastante desenvolvido; razoável situação financeira dos órgãos públicos; cultura organizacional; 
$\checkmark$ Ameaças: Extensão (profissões afins atuando na área contábil); atuação do Ministério Público por meio das restrições impostas às fundações;

$\checkmark$ Fatores críticos de sucesso: Corpo docente, servidores técnico-administrativos, coordenação, discentes, egressos, organizações, concorrentes e MEC.

Com base nas informações contidas no planejamento estratégico, pode-se estruturar o Balanced Scorecard para a unidade em estudo, ou seja, traduz-se a visão estratégica do departamento de Ciências Contábeis da UFSC em objetivos e medidas capazes de serem avaliadas e comparadas, ao fornecer uma estrutura de comunicação da visão estratégica para todos os funcionários (KAPLAN; NORTON, 1992; CAMPBELL, 1997; TSENG, 2010).



Figura 2 Subáreas do departamento de Ciências Contábeis da UFSC Fonte: Planejamento estratégico 2009-2020 (CCN, 2011c).

Cabe destacar que, conforme apresentado anteriormente, a maioria dos estudos relacionados à temática BSC utilizam quatro perspectivas de análise: financeira, clientes, processos internos e aprendizagem e crescimento. No entanto, para os fins deste estudo, optase por trabalhar com abordagens distintas. As abordagens são classificadas em perspectivas e subáreas, sendo compostas por pessoas (corpo docente e técnico-administrativo), processos (coordenação), clientes (discentes e egressos) e sociedade (organizações, concorrentes e MEC).

O mapa estratégico é um quadro visual para os objetivos definidos dentro das áreas ou perspectivas. Esses mapas mostram como uma organização faz planos para converter seus vários ativos em resultados desejados. Os mapas estratégicos fornecem aos funcionários uma forma de visão clara de como seus esforços estão ligados aos objetivos globais da organização, permitindo-lhes trabalhar de forma coordenada e colaborativa em direção aos objetivos desejados pela organização. Sugerem-se dois passos para a construção do mapa: 1) 


\section{O BALANCED SCORECARD COMO FERRAMENTA DE GESTÃO ESTRATÉGICA EM UMA UNIVERSIDADE FEDERAL \\ DOI: http://dx.doi.org/10.5007/1983-4535.2012v5n3p1}

compreensão dos objetivos estratégicos das organizações; 2) tradução dos objetivos para as perspectivas de trabalho definidas (KAPLAN; NORTON, 2000).

Neste estudo, o mapa estratégico utilizado no processo de elaboração do BSC é uma estrutura adaptada para descrever a estratégia sob perspectivas consideradas relevantes pela organização, explicitando-se um conjunto de objetivos conectados por hipóteses da estratégia. Assim, foram definidos 26 objetivos para compreender as perspectivas definidas conforme estrutura apresentada no mapa estratégico (figura 3).

O mapa está estruturado em quatro perspectivas, e estas são divididas em sete subáreas. A base do planejamento do departamento está focada nas pessoas, ou seja, no corpo docente e nos servidores técnico-administrativos. Estas, por meio da coordenação, interagem com o corpo discente e com os egressos. Por fim, no topo do mapa encontra-se a sociedade. Esse grupo é formado por organizações, concorrentes e MEC.

O processo de gestão pressupõe as atividades de planejamento, execução e controle. O planejamento foi desenvolvido pelo departamento de Contabilidade, do qual resultaram os objetivos dispostos nas áreas apresentadas no mapa estratégico. Portanto, a etapa posterior se refere ao detalhamento do plano, tendo em vista a sua implementação e a sua execução. É imprescindível, nesse momento, que o que, como, quando, quanto, onde e quem estejam bem definidos. Não se concebe a ideia de que a execução do plano seja realizada antes ou mesmo simultaneamente a esse detalhamento, pois isso pode acarretar em turbulências, desencontros e atrasos (BARROS, 2005).

Segundo Kaplan e Norton (2008), o mau desempenho da maioria das empresas é devido a discrepâncias entre estratégia e operações. Assim, a partir da visão e estratégia da empresa, são traçadas metas para cada perspectiva e, para cada meta, são traçados indicadores para avaliar o seu desempenho. Esses indicadores são responsáveis pelo acompanhamento das estratégias, o que minimiza as discrepâncias mencionadas por Kaplan e Norton (2008) (FREITAS et al., 2010).

Nesse contexto, as fases de execução e controle atendem os planos aprovados e verificam se eles atingem os objetivos esperados. Nessas fases do processo de gestão, a atenção deve ser dada à efetiva realização das operações previstas. É nessa fase que se consolida a eficácia das atividades (CROZATTI, 2003). Assim, apresenta-se na figura 4 o painel balanceado que contém os objetivos (planejamento) e os indicadores (controle) de cada 


\section{O BALANCED SCORECARD COMO FERRAMENTA DE GESTÃO ESTRATÉGICA EM UMA \\ UNIVERSIDADE FEDERAL}

DOI: http://dx.doi.org/10.5007/1983-4535.2012v5n3p1

subárea. Posteriormente, apresentam-se as ações (execução) para colocá-los em prática, as metas estipuladas e a medida de referência.

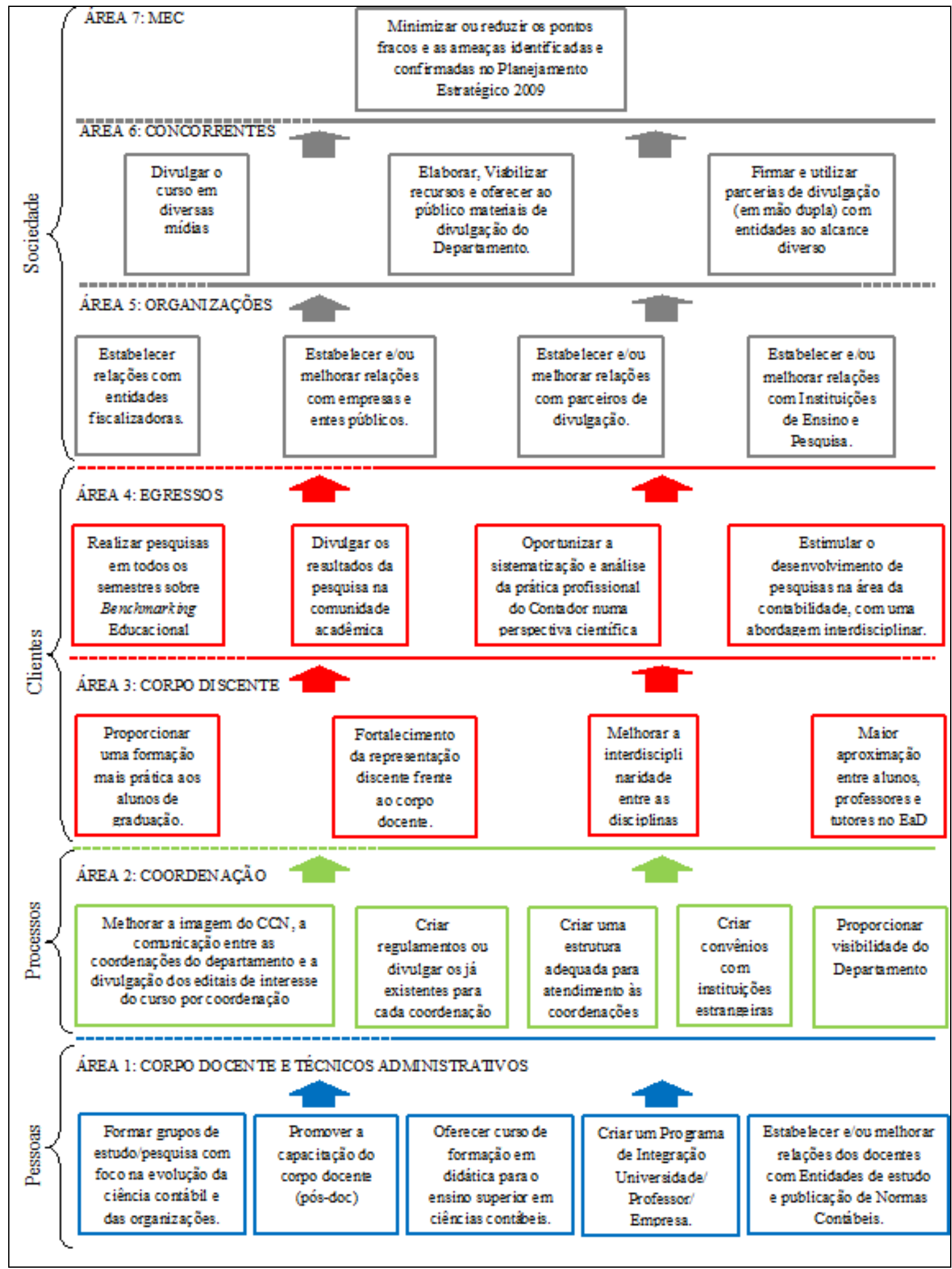

Figura 3 Mapa estratégico

Fonte: Planejamento estratégico 2009-2020 (CCN, 2011c). 


\section{O BALANCED SCORECARD COMO FERRAMENTA DE GESTÃO ESTRATÉGICA EM UMA UNIVERSIDADE FEDERAL \\ DOI: http://dx.doi.org/10.5007/1983-4535.2012v5n3p1}

\begin{tabular}{|c|c|c|c|}
\hline Perspectiva & Sub-área & Objetivos & Indicadores \\
\hline \multirow{5}{*}{ Pessoas } & \multirow{5}{*}{$\begin{array}{l}\text { CORPO DOCENTE E } \\
\text { TÉCNICOS } \\
\text { ADMINISTRATIVOS }\end{array}$} & Formar grupos de estudo/pesquisa & $\begin{array}{c}\% \text { de professores com grupos de estudos } \\
\text { registrados no CNPQ }\end{array}$ \\
\hline & & $\begin{array}{l}\text { Promover a capacitação do corpo docente } \\
\text { (pós-doc) }\end{array}$ & $\begin{array}{c}\% \text { de doutores com Pós-Doc concluído ou } \\
\text { em desenvolvimento }\end{array}$ \\
\hline & & $\begin{array}{c}\text { Oferecer curso de formação em didática } \\
\text { para o ensino superior em ciências } \\
\text { contábeis. }\end{array}$ & $\begin{array}{l}\% \text { de docentes que cursaram ao menos um } \\
\text { curso por ano dos ofericidoa pelo Profor. }\end{array}$ \\
\hline & & $\begin{array}{l}\text { Criar programas de Integração } \\
\text { Universidade/ Professor/ Empresa. }\end{array}$ & Número de programas \\
\hline & & $\begin{array}{c}\text { Estabelecer relações dos docentes com } \\
\text { Entidades de estudo e publicação de } \\
\text { Normas Contábeis. }\end{array}$ & $\begin{array}{l}\% \text { de professores pertencentes ao CFC, } \\
\text { CPC, Ibracon, CRCs }\end{array}$ \\
\hline \multirow{5}{*}{ Processos } & \multirow{5}{*}{ COORDENAÇÃO } & $\begin{array}{l}\text { Melhorar a comunicação entre as } \\
\text { coordenações do departamento }\end{array}$ & $\begin{array}{l}\text { Regulamentos aprovados/total de } \\
\text { coordenações }\end{array}$ \\
\hline & & $\begin{array}{c}\text { Criar Parcerias de Integração } \\
\text { Universidade/ Profess or/ Empresa. }\end{array}$ & Número de parcerias (extensão e pesquisa) \\
\hline & & $\begin{array}{l}\text { Oferecer estrutura adequada para } \\
\text { atendimento às coordenações }\end{array}$ & $\begin{array}{l}\text { Coordenadorias com espaço próprio para } \\
\text { atendimento/Número de coordenadorias }\end{array}$ \\
\hline & & $\begin{array}{l}\text { Criar convênios com instituições } \\
\text { estrangeiras }\end{array}$ & Número de convênios criados \\
\hline & & $\begin{array}{l}\text { Proporcionar vis ibilidade do } \\
\text { Departamento }\end{array}$ & $\begin{array}{l}\text { Número de políticas desenvolvidas que } \\
\text { propiciam visibilidade ao departamento }\end{array}$ \\
\hline \multirow{8}{*}{ Clientes } & \multirow{4}{*}{ CORPO DISCENTE } & $\begin{array}{l}\text { Proporcionar uma formação prática aos } \\
\text { alunos de graduação. }\end{array}$ & $\begin{array}{c}\text { Horas de disciplinas práticas/Horas totais } \\
\text { do curso }\end{array}$ \\
\hline & & $\begin{array}{l}\text { Fortalecimento da representação discente } \\
\text { frente ao corpo docente. }\end{array}$ & $\begin{array}{c}\text { Aumentar a proporção de direito à voto do } \\
\text { CACIC nas decisões do colegiado }\end{array}$ \\
\hline & & $\begin{array}{l}\text { Melhorar a interdisciplinaridade entre as } \\
\text { disciplinas }\end{array}$ & $\begin{array}{l}\text { Número de reuniões entre as disciplinas } \\
\text { afins por semestre }\end{array}$ \\
\hline & & $\begin{array}{c}\text { Maior aproximação entre alunos, } \\
\text { professores e tutores no EaD }\end{array}$ & Número de reuniões mensais \\
\hline & \multirow{4}{*}{ EGRESSOS } & $\begin{array}{c}\text { Realizar pesquisas sobre Benchmarking } \\
\text { Educacional }\end{array}$ & Número de pesquisas realizadas por ano \\
\hline & & $\begin{array}{c}\text { Divulgar os resultados da pesquis a na } \\
\text { comunidade acadêmica }\end{array}$ & $\begin{array}{c}\text { Número de fontes nas quais as pesquisas } \\
\text { estão disponíveis }\end{array}$ \\
\hline & & $\begin{array}{c}\text { Oportunizar a sistematização e análise da } \\
\text { prática profissional do Contador numa } \\
\text { perspectiva científica }\end{array}$ & $\%$ de artigos derivados de TCCs \\
\hline & & $\begin{array}{c}\text { Estimular o desenvolvimento de pesquisas } \\
\text { na área da contabilidade, com uma } \\
\text { abordagem interdisciplinar. }\end{array}$ & $\begin{array}{l}\% \text { de egressos que publicam com } \\
\text { docentes do } \mathrm{CCN}\end{array}$ \\
\hline \multirow{8}{*}{ Sociedade } & \multirow{4}{*}{ ORGANIZAÇÕES } & $\begin{array}{l}\text { Estabelecer relações com entidades } \\
\text { fiscalizadoras. }\end{array}$ & $\begin{array}{l}\% \text { de professores que pertencem a estas } \\
\text { entidades fiscalizadoras ou reguladoras }\end{array}$ \\
\hline & & $\begin{array}{l}\text { Melhorar relações com empresas e entes } \\
\text { públicos. }\end{array}$ & $\begin{array}{l}\text { Número de convênios com empresas e } \\
\text { entes públicos }\end{array}$ \\
\hline & & $\begin{array}{c}\text { Estabelecer relações com parceiros de } \\
\text { divulgação. }\end{array}$ & Participações na TV UFSC/TVCRC/TVCFC \\
\hline & & $\begin{array}{l}\text { Estabelecer e/ou melhorar relações com } \\
\text { Instituições de Ensino e Pesquisa. }\end{array}$ & $\begin{array}{l}\text { Número de de bolsas (pibic, capes, reuni, } \\
\text { PQ) }\end{array}$ \\
\hline & \multirow{3}{*}{ CONCORRENTES } & Divulgar o curso em diversas mídias & Número de mídias \\
\hline & & $\begin{array}{c}\text { Elaborar, Viabilizar recursos e oferecer ao } \\
\text { público materiais de divulgação do } \\
\text { Departamento. }\end{array}$ & $\begin{array}{l}\text { Números diferentes de materias de } \\
\text { divulgação distribuidos }\end{array}$ \\
\hline & & $\begin{array}{c}\text { Firmar e utilizar parcerias de divulgação } \\
\text { (em mão dupla) com entidades ao alcance } \\
\text { diverso }\end{array}$ & $\begin{array}{l}\text { Número de entidades que existe parceria } \\
\text { efetiva (documental) }\end{array}$ \\
\hline & MEC & $\begin{array}{c}\text { Minimizar ou reduzir os pontos fracos e as } \\
\text { ameaças identificadas e confirmadas no } \\
\text { Planejamento Es tratégico } 2009\end{array}$ & $\begin{array}{c}\% \text { de pontos fracos detectados no PE que } \\
\text { foram reduzidos }\end{array}$ \\
\hline
\end{tabular}

Figura 4 Painel balanceado, parte 1

Fonte: Adaptado do planejamento estratégico 2009-2020 (CCN, 2011c). 


\section{O BALANCED SCORECARD COMO FERRAMENTA DE GESTÃO ESTRATÉGICA EM UMA \\ UNIVERSIDADE FEDERAL \\ DOI: http://dx.doi.org/10.5007/1983-4535.2012v5n3p1}

Conforme se pode observar na figura 4, a parte inicial do painel está disposta em quatro colunas, sendo a primeira da perspectiva, a segunda das subáreas, a terceira dos objetivos e a quarta dos indicadores definidos para medir e avaliar a situação dos objetivos. Contudo, para que a implementação do BSC possa trazer resultados positivos para a organização, todos os colaboradores devem tomar iniciativas para atingir as metas estipuladas. Para os fins deste estudo, no sentido de preservar a estratégia do departamento, são expostas, na figura 5, apenas as informações estratégicas detalhadas da perspectiva "pessoas".

\begin{tabular}{|c|c|c|c|c|}
\hline Objetivos & Indicadores & Referência & Meta & Ações/Iniciativas \\
\hline Formar grupos de estudo/pesquisa & $\begin{array}{l}\% \text { de professores com grupos de estudos } \\
\text { registrados no CNPQ }\end{array}$ & $25 \%$ & $50 \%$ & $\begin{array}{c}\text { Conscientizar os docentes a respeito da } \\
\text { importância do desenvolvimento das } \\
\text { pesquisas }\end{array}$ \\
\hline $\begin{array}{l}\text { Promover a capacitação do corpo docente } \\
\text { (pós-doc) }\end{array}$ & $\begin{array}{c}\% \text { de doutores com Pós-Doc concluído ou } \\
\text { em desenvolvimento }\end{array}$ & $20 \%$ & $50 \%$ & $\begin{array}{c}\text { Ciar parcerias com universidades } \\
\text { estrangeiras para possibilitar o Pós-Doc } \\
\text { dos docentes }\end{array}$ \\
\hline $\begin{array}{l}\text { Oferecer curso de formação em didática } \\
\text { para o ensino superior em ciências } \\
\text { contábeis. }\end{array}$ & $\begin{array}{l}\% \text { de docentes que cursaram ao menos um } \\
\text { curso por ano dos ofericidos pelo Profor. }\end{array}$ & $22 \%$ & $80 \%$ & $\begin{array}{l}\text { Exigir \% de participação em cursos para } \\
\text { progressão funcional }\end{array}$ \\
\hline $\begin{array}{l}\text { Criar programas de Integração } \\
\text { Universidade/ Professor/ Empresa. }\end{array}$ & Número de programas & 2 & 5 & $\begin{array}{c}\text { Criar um canal de comunicação } \\
\text { professor/empresa por meio de fundações } \\
\text { de apoio }\end{array}$ \\
\hline $\begin{array}{l}\text { Estabelecer relações dos docentes com } \\
\text { Entidades de estudo e publicação de } \\
\text { Normas Contábeis. }\end{array}$ & $\begin{array}{c}\% \text { de professores pertencentes ao CFC, } \\
\text { CPC, Ibracon, CRCs }\end{array}$ & $15 \%$ & $30 \%$ & $\begin{array}{c}\text { Incentivar a participação de docentes } \\
\text { nestas entidades }\end{array}$ \\
\hline
\end{tabular}

Figura 5 Painel balanceado, parte 2 - Pessoas

Fonte: Elaborado pelos autores.

$\mathrm{Na}$ figura 5 apresentam-se, além dos objetivos e indicadores da perspectiva de pessoas já demonstrados anteriormente, as metas estipuladas para o departamento, as ações/iniciativas que devem ser tomadas para o alcance de tais metas, bem como a situação atual (referência) dos indicadores.

Em relação ao primeiro indicador (percentual de professores com grupos de estudos registrados no $\mathrm{CNPq}$ ) tem-se que, na unidade de estudo, $25 \%$ dos docentes possuem grupos de pesquisa registrados. Contudo, tomando-se como parâmetro a Universidade de São Paulo (USP), que é considerada uma das melhores universidades do país e que possui, atualmente, $40 \%$ de seus docentes com grupos de pesquisa registrados, estima-se como meta para o departamento de Ciências Contábeis da UFSC o percentual de 50\% até 2020 .

Para o indicador de percentual de doutores com pós-doutorado concluído ou em desenvolvimento, tem-se como referência $20 \%$ dos docentes. Pretende-se, até 2020 , que $50 \%$ dos doutores possuam pós-doutorado. Para isso, tem-se como iniciativa criar parcerias com universidades estrangeiras para possibilitar tal formação. Cabe destacar que, atualmente, a USP possui $25 \%$ dos seus doutores com pós-doutorado. 


\section{O BALANCED SCORECARD COMO FERRAMENTA DE GESTÃO ESTRATÉGICA EM UMA UNIVERSIDADE FEDERAL \\ DOI: http://dx.doi.org/10.5007/1983-4535.2012v5n3p1}

Os três indicadores que completam a perspectiva de pessoas (percentual de docentes que cursaram ao menos um curso por ano dos oferecidos pelo Profor, número de programas e percentual de professores pertencentes ao CFC, CPC, Ibracon, CRCs) têm metas definidas internamente, ou seja, não seguem o padrão dos indicadores anteriores, que possuíam como referência a USP. Nesse caso, as metas foram definidas levando-se em consideração as perspectivas e as limitações do departamento.

Cabe destacar que, para que o departamento de Ciências Contábeis da UFSC possa atingir sua missão e visão - respectivamente, "produzir e difundir os conhecimentos da área contábil, através do ensino, pesquisa e extensão, formando profissionais qualificados e, complementarmente, o cidadão como elemento que contribua para o desenvolvimento e a melhoria da qualidade de vida da sociedade" e "ser o melhor curso de ciências contábeis do Brasil" (CCN, 2011c) -, todas as metas estipuladas precisam ser atingidas. Assim, deve haver um acompanhamento contínuo, tendo em vista a detecção de possíveis falhas de planejamento ou até mesmo uma mudança na conjuntura econômica que possa afetar o desempenho do departamento. Nessa ótica, Campbell (1997) afirma que o BSC reflete o plano estratégico da organização em um quadro que permite a cada pessoa medir seu desempenho e efetuar ajustes imediatos, se necessário, com vistas ao aprimoramento contínuo.

\section{CONCLUSÕES}

A avaliação de desempenho de uma universidade é um processo contínuo, o qual requer monitoramento constante para que se mantenha um alto nível de controle dos processos que interferem numa série de aspectos de uma organização. No entanto, ainda existem poucos estudos sobre o desenvolvimento e a implementação do BSC para medir o desempenho das atividades de universidades (TSENG, 2010). Neste estudo, para contribuir com o processo de gestão do departamento de Ciências Contábeis da UFSC, utiliza-se a ferramenta Balanced Scorecard, que pode auxiliar a gestão estratégica de duas maneiras complementares: 1) identificar e implementar uma visão estratégica para os objetivos estratégicos e operacionais; 2) facilitar o processo de gestão estratégica (PIENAAR; PENZHORN, 2000).

O modelo utilizado é derivado dos estudos de Kaplan e Norton (1992). Para a fundamentação do tema utiliza-se de duas correntes de autores: a primeira trabalha com as quatro perspectivas (clientes, financeira, processos internos, aprendizagem e crescimento) 


\section{O BALANCED SCORECARD COMO FERRAMENTA DE GESTÃO ESTRATÉGICA EM UMA UNIVERSIDADE FEDERAL \\ DOI: http://dx.doi.org/10.5007/1983-4535.2012v5n3p1}

definidas inicialmente por Kaplan e Norton; a segunda adapta essas perspectivas à organização objeto dos seus estudos. Assim, neste artigo, utilizam-se perspectivas adaptadas, que por sua vez são divididas em subáreas (figura 3).

Para a elaboração do BSC, parte-se das informações contidas no planejamento estratégico do departamento, desenvolvido para o período 2009-2020. Com base nessas informações, identificaram-se as perspectivas de atuação e suas respectivas subáreas. Com isso, elaborou-se o mapa estratégico do departamento, a fim de estruturar os objetivos da unidade de negócio em estudo.

O mapa estratégico contempla quatro perspectivas, sete subáreas e vinte e seis objetivos, bem como as relações entre eles. A base do mapa são as pessoas, que por meio de processos se relacionam com os clientes e com a sociedade. Posteriormente, com base nos objetivos definidos, elaborou-se o painel balanceado. Nele, cada objetivo possui um indicador de controle, um valor de referência, uma meta e ações ou iniciativas a serem tomadas para atingir tal objetivo. No entanto, por serem informações estratégicas, foram apresentadas de forma mais detalhada apenas as informações da perspectiva "pessoas".

Cabe destacar que, para o curso de Ciências Contábeis da Universidade Federal de Santa Catarina "ser o melhor curso de ciências contábeis do Brasil", todas as metas estipuladas precisam ser atingidas, já que atualmente (referência na figura 5) os indicadores apontam valores menores que os da instituição utilizada como parâmetro - nesse caso, a USP. Para se atingir a situação almejada, deve haver, ainda, um acompanhamento contínuo por parte dos "gestores do BSC" - nesse caso, na função de coordenadores de subáreas -, para que as ações sejam, efetivamente, colocadas em prática.

Ao se fazer uma relação com a metodologia desenvolvida por Papalexandris et al. (2005) para a implantação de um BSC, cujas fases são I) preparar para o projeto, II) compreender a visão e estratégia, III) identificar as prioridades estratégicas e objetivos, IV) selecionar medidas de desempenho, V) operacionalizar o projeto e VI) implementar e acompanhar o sistema, percebe-se que este artigo cumpriu as quatro primeiras etapas. As etapas V e VI fogem ao controle dos autores, e fica a cargo do departamento de Ciências Contábeis da UFSC operacionalizar e acompanhar o projeto.

Nessa ótica, sugere-se para futuros trabalhos o acompanhamento da execução do BSC desenvolvido neste trabalho, tendo em vista que os indicadores aqui definidos precisam ser validados para que haja o alinhamento entre a estratégia e a sua operacionalização. Além 
disso, realizando-se um acompanhamento contínuo, as metas podem ser ajustadas, indicadores e objetivos podem ser repensados e novas iniciativas podem ser criadas.

\section{REFERÊNCIAS}

AX, Christian; BJORNENAK, Trond. Bundling and diffusion of management accounting innovations: the case of the balanced scorecard in Sweden. Management Accounting Research, v. 16, p. 1-20, 2005.

BARROS, Vaine de Magalhães. O novo velho enfoque da informação contábil. Revista Contabilidade \& Finanças, v. 38, p. 102-112, 2005.

BOURGUIGNON, Annick; MALLERET, Véronique; NORREKLIT, Hanne. The American balanced scorecard versus the French tableau de bord: the ideological dimension.

Management Accounting Research, v. 15, p. 107-134, 2004.

BRAAM, Geert J. M.; NIJSSEN, Edwin J. Performance effects of using the Balanced Scorecard: a note on the Dutch experience. Long Range Planning, v. 37, p. 335-349, 2004.

CAMPBELL, A. Keeping the engine humming. Business Quarterly Summer, p. 40-46. 1997.

CCN - Departamento de Ciências Contábeis da Universidade Federal de Santa Catarina. Site institucional. 2011a. Disponível em: <www.ccn.ufsc.br>. Acesso em: 5 set. 2011.

Currículo do curso de ciências contábeis 2006-1. 2011b. Disponível em:

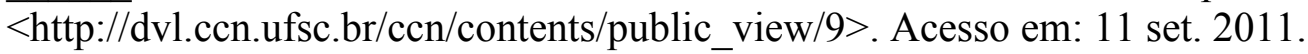
$2011 c$.

Planejamento estratégico do curso de ciências contábeis UFSC 2009-2020.

CHAVAN, Meena. The balanced scorecard: a new challenge. Journal of Management Development, v. 28, p. 393-406, 2009.

CHEN, Mu-Yen; HUANG, Mu-Jung; CHENG, Yu-Chen. Measuring knowledge management performance using a competitive perspective: an empirical study. Expert Systems with Applications, v. 36, p. 8449-8459, 2009.

CHIAVENATO, Idalberto. Introdução à teoria geral da administração. 7. ed. rev. e atual. Rio de Janeiro: Elsevier, 2003.

CROZATTI, Jaime. Planejamento estratégico e controladoria: um modelo para potencializar a contribuição das áreas da organização. ConTexto, v. 3, n. 5, 2003.

DAVIS, Stan; ALBRIGHT, Tom. An investigation of the effect of Balanced Scorecard implementation on financial performance. Management Accounting Research, v. 15, p. 135-153, 2004. 


\section{O BALANCED SCORECARD COMO FERRAMENTA DE GESTÃO ESTRATÉGICA EM UMA \\ UNIVERSIDADE FEDERAL \\ DOI: http://dx.doi.org/10.5007/1983-4535.2012v5n3p1}

DIETSCHI, Daniel A.; NASCIMENTO, Auster M.. Um estudo sobre a aderência do Balanced Scorecard às empresas abertas e fechadas. Revista Contabilidade e Finanças, v. 19, n. 46, p. 73-85, 2008.

FREITAS, M. M. M. et al. O Balanced Scorecard em entidades sem fins lucrativos: um estudo de caso. In: CONGRESSO BRASILEIRO DE CUSTOS, 17., 2010, Belo Horizonte. Anais... Belo Horizonte: CBC, 2010.

GALAS, Eduardo S.; PONTE, Vera M. R.. O Balanced Scorecard e o alinhamento estratégico da tecnologia da informação: um estudo de casos múltiplos. Revista Contabilidade e Finanças, n. 40, p. 37-51, 2006.

GIL, Antonio C.. Como elaborar projetos de pesquisa. 4. ed. São Paulo: Atlas, 2007.

GLAT, Rosana; PLETSCH, Márcia D. O papel da universidade frente às políticas públicas para educação inclusiva. Revista Benjamim Constant, ano 10, n. 29, p. 3-8, 2004.

GUMBUS, Andra; LUSSIER, Robert N. Entrepreneurs using a balanced scorecard to translate strategy into objectives and measures. Journal of Small Business Management, v. 44, p. 407-425, 2006.

HSIEH, Ling-Feng; CHIN, Jiung-Bin; WU, Mu-Chen. The performance indicators of university e-library in Taiwan. The Electronic Library, v. 22, n. 4, p. 325-330, 2004.

KALD, M.; NILSSON, F. Performance measurement at Nordic companies. European Management Journal, v. 1, p. 113-127, 2000.

KAPLAN, R. S.; NORTON, D. P. The balanced scorecard: measures that drive performance. Harvard Business Review, p. 71-79, jan./fev. 1992.

. A estratégia em ação: balanced scorecard. 5. ed. Rio de Janeiro: Campus, 1997.

2000.

Having trouble with your strategy? then map it. Harvard Business Review, set./out.

Mastering the management system. Harvard Business Review, jan. 2008.

KAPLAN, R. S.; NORTON, D. P; RUGELSJOEN, B. Managing alliances with the balanced scorecard. Harvard Business Review, jan./fev. 2010.

KELM, M. L. Indicadores de performance em uma instituição universitária autogerida: uma contribuição à gestão por resultados. Tese (Doutorado em Engenharia de Produção) Universidade Federal de Santa Catarina. Florianópolis, 2003.

KONGAR, Elif; PALLIS, Jani M.. Non-parametric approach for evaluating the performance of engineering schools. International Journal of Engineering, v. 26, n. 5, p. 1210-1219, 2010. 


\section{O BALANCED SCORECARD COMO FERRAMENTA DE GESTÃO ESTRATÉGICA EM UMA UNIVERSIDADE FEDERAL \\ DOI: http://dx.doi.org/10.5007/1983-4535.2012v5n3p1}

LIPEA, Marlys G.; SALTERIO, Steven. A note on the judgmental effects of the balanced scorecard's information organization. Accounting, Organizations and Society, v. 27, p. 531$540,2002$.

MARTINSONS, Maris; DAVISON, Robert; TSE, Dennis. The balanced scorecard: a foundation for the strategic management of information systems. Decision Support Systems, v. 25, p. 71-88, 1999.

OTLEY, David. Performance management: a framework for management control systems research. Management Accounting Research, v. 10, p. 363-382, 1999.

PAPALEXANDRIS, Alexandros; IOANNOU, George; PRASTACOS, Gregory P. Implementing the Balanced Scorecard in Greece: a software firm's experience. Long Range Planning, v. 37, p. 351-366, 2004.

PAPALEXANDRIS, Alexandros et al. An integrated methodology for putting the Balanced Scorecard into action. European Management Journal, v. 23, p. 214-227, 2005.

PESSOA, M. N. M. Gestão das universidades federais brasileiras: um modelo fundamentado no balanced scorecard. Tese (Doutorado) - Universidade Federal de Santa Catarina, Centro Tecnológico. Florianópolis, 2000. 343 p.

PIENAAR, Heila; PENZHORN, Cecilia. Utilizando o balanced scorecard para facilitar a gestão estratégica em um serviço de informações acadêmicas. Libri, v. 50, p. 202-209, 2000.

RABBANI, Fauziah et al. Designing a balanced scorecard for a tertiary care hospital in Pakistan: a modified Delphi group exercise. International Journal of Health Planning and Management, v. 25, p. 74-90, 2010.

ROCHA, Douglas J. A.. Desenvolvimento do balanced scorecard para instituição de ensino superior privada: estudo de caso da unidade de negócios 4 da universidade gama. Dissertação (Mestrado em Engenharia de Produção) - Universidade Federal de Santa Catarina. Florianópolis, 2000.

SOUZA, Antonio C.; FIALHO, Francisco A. P.; OTANI, Nilo. TCC: métodos e técnicas. Florianópolis: Visual Books, 2007.

SPECKBACHER, Gerhard; BISCHOF, Juergen; PFEIFFER, Thomas. A descriptive analysis on the implementation of Balanced Scorecards in German-speaking countries. Management Accounting Research, v. 14, p. 361-387, 2003.

TRIVIÑOS, Augusto N. S.. Introdução à pesquisa em ciências sociais: a pesquisa qualitativa em educação. São Paulo: Atlas, 1990.

TSENG, Ming-Lang. Implementation and performance evaluation using the fuzzy network balanced scorecard. Computers \& Education, v. 55, p. 188-201, 2010. 
UFSC - Universidade Federal de Santa Catarina. Plano de desenvolvimento institucional. 2011. Disponível em: < http://pdi.paginas.ufsc.br/>. Acesso em: 25 ago. 2011.

WACHTEL, T. L; HARTFORD, C. E.; HUGHES, J. A. Building a Balanced Scorecard for a burn center. Burns, v. 25, p. 431-437, 1999.

YU, May Leen et al. The e-balanced scorecard (e-BSC) for measuring academic staff performance excellence. High Educ., v. 57, p. 813-828, 2009.

ZBINDEN, Alex M. Introducing a balanced scorecard management system in a university anesthesiology department. Anesth. Analg., v. 95, p. 1731-1738, 2002. 